\title{
ENHANCING ACOUSTIC SENSORY RESPONSIVENESS BY EXPLOITING BIO-INSPIRED FEEDBACK COMPUTATION
}

\author{
José Guerreiro, Joseph C. Jackson and James F. C. Windmill \\ Centre for Ultrasonic Engineering, University of Strathclyde, Glasgow, Scotland, U.K. \\ \{jose.guerreiro, joseph.jackson, james.windmill\}@ strath.ac.uk
}

\begin{abstract}
Engineering acoustic sensors and systems that can be sensitive to small sound levels even when immersed by background noise may require out-of-the-box thinking. Biology can provide inspiration for that, allowing the engineering landscape to borrow interesting ideas and thus solve current human problems. Biological sensor and system designs are a result of many million years of evolutionary processes, which make them very-power efficient and welladapted to perform their function in a living organism. This paper presents a theoretical study of a bio-inspired signal processing concept. The assumption is that by exploiting feedback computation between a front-end acoustic detector and a back-end neuronal based processing, the overall acoustic responsiveness of a sensory system can be controlled and enhanced to target signals of interest. Here, two methods of feedback signal entrainment are compared namely 1:1 and 2:1 resonance modes.
\end{abstract}

Index Terms - bio-inspired acoustic sensor system, adaptive signal processing, nonlinear system dynamics, feedback computation, neuronal model.

\section{INTRODUCTION}

Past studies have extrapolated that biological sensors and systems exhibit passive and active mechanisms performed at early states of the signal processing chain, which are thought to be of great benefit in terms of signal-detection capabilities and cost of computation [1-2]. For instance, the peripheral auditory system is designed with a type of analogue-digital feedback based system architecture able to accomplish optimized signal conditioning and processing tasks. Including selective filtering (e.g., tuning) and active nonlinear amplification before signals are sampled and compressed by the neuronal system in a form of (bio)electrical pulses (e.g., action potentials) [3-4]. Motivated by that, this paper presents a modeling approach for a potential bio-inspired sensor system concept applied to acoustics. The aim is to present an interesting signal processing framework applied at the transducer level, which might provide some potential benefits to the acoustic engineering namely applied to enhance signal-detection tasks.

\subsection{Passive Sensory Responses}

A simple and well-known approach to model the passive resonant acousto-mechanical responses of a sound detector (e.g., acoustic transducer), either biological- or man-made, is through a single degree of freedom mass-spring system that exhibits a $2^{\text {nd }}$ order response [5], which in Laplace form can be expressed as the transfer function in:

$$
H(s)=\frac{s \frac{\omega_{0}}{Q}}{s^{2}+s \frac{\omega_{0}}{Q}+\omega_{0}^{2}}
$$

where $s$ is the Laplace term $(s=i \omega)$, $\omega_{0}$ represents the resonance frequency of the system and $Q$ its quality factor. Normally, the sensitivity of a front-end acoustic detector is greatest when operated at its resonance frequency, and that might be approximated to a bandpass filter response with bandwidth proportionally dependent on the $Q$-factor. The $Q$ factor expresses how damped the system is, and as such an overdamped system may exhibit a low $Q$-factor (e.g., faster time response, and wider bandwidth) and an underdamped system a high $Q$-factor (e.g., slower time response, and narrower bandwidth), respectively.

\subsection{Active Sensory Responses}

Conventional transducer design techniques may set physical constraints on the sensor or system responses, which thus result in limited or static values of the $Q$-factor and $\omega_{0}$ for a desired front-end acoustic system response. However, studies have already shown that the $Q$-factor of some sensors can be manipulated through the use of feedback control mechanisms [6-7], and that the $\omega_{0}$ can also be changed dynamically by exploiting feedback system approaches in a similar manner [8]. Interestingly, some biological sensors and systems such as those involved in the process of hearing, are thought to operate with feedback control techniques in order to dynamically adapt their front-end acoustic responses - the process known as active hearing [9-10]. For instance, the mosquito's auditory system provides a good example, since it is thought to exhibit some sort of fast-feedback adaptation process, operating on a cycle-by-cycle basis [11]. The assumption is the following: the active hearing responses of such an auditory system are thought to be greatly enhanced through synchronized neurons adding extra energy to the front-end acoustic mechanical detector (e.g., antenna). As a 
consequence, nonlinearities may result from this unconventional method of signal detection such as compressive gain, hysteretic output responses and generation of intermodulation products may result. These are rather be considered as interesting sensor system responses, which are empowered by a positive-controlled feedback system architecture that is designed to achieve fast-adaptation and enhanced sensory responsiveness to target input signals of interest. Some degree of thought may support the fact that these active hearing processes might be originated from a kind of critical-system [12]. If that is true, it can be a reason for signals to be quickly amplified, and in a nonlinear fashion, and therefore with great benefit for a signal-detection task, as illustrated in Fig. 1.

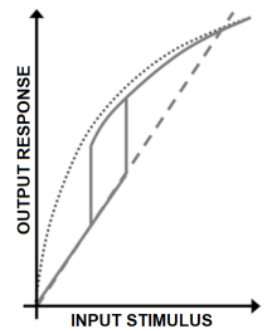

Fig. 1 - Example of active hearing responses. (Dash-line) represents a system or detector with a linear response (e.g., passive response), such as a driven harmonic oscillator $-2^{\text {nd }}$ order response; (dot-line) is a typical vertebrate hair bundle nonlinear response (e.g., active response); (solid-line) is a hysteretic nonlinear response such as reported from the mosquito hearing system (e.g., active response). Image adapted and redrawn from [13].

A modeling approach to study a bio-inspired feedback sensor system architecture using a front-end analogue detector and a back-end digital computational method is presented as follows.

\section{ADAPTIVE SOUND PROCESSING}

Motivated by the active mechanisms of signal-detection and processing within biological sensors and systems, past studies have presented novel sensor system architectures that exploit a bio-inspired neuronal model approach namely using the leaky-integrate-and-fire (LIF) neuron [14-15]. It led to the creation of a novel design approach for an acoustic signal processing methodology performed at the transducer level [16-17].

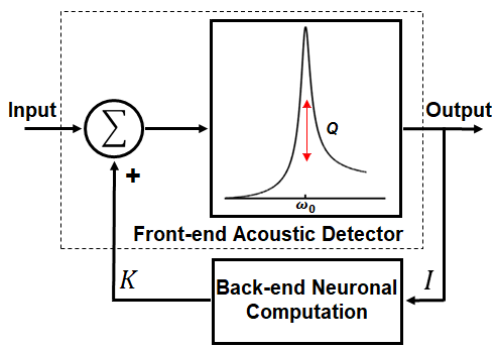

Fig. 2 - Diagram overview of the feedback control system that is used to implement the concept of adaptive sound processing. Image adapted and redrawn from [16].
This can be modelled using a feedback control process approach using a mechanical detector (e.g., analogue function) equipped with some sort of electrical computation capabilities (e.g., digital functions) that together can perform peripheral sound processing, hence the "transducer can be part of the signal processing chain", as illustrated in Fig. 2. Here, "Front-end Acoustic Detector" represents a typical sensory response of an analogue acoustic detector as a $2^{\text {nd }}$ order system (refer to (1)); and "Back-end Neuronal Computation" represents a digital feedback control process that is based on the LIF model system, computing signals in a cycle-by-cycle manner. The LIF function (refer to (2)) is used as a control mechanisms and it is meant to inject additional energy $(K)$ to the front-end acoustic detector according to the level of past signals detected $(I)$. The biological reports that inspire this concept are based on the mosquito nonlinear hearing responses being greatly enhanced due to the "synchrony through twice-frequency forcing" - 2:1 resonance mode as presented in Fig. 3 (B), in (A) the 1:1 resonance mode is shown for comparison. It means that $1: 1$ mode provides entrainment of pulses (e.g., feedback signals) once per input-cycle, whereas, 2:1 mode provides entrainment of pulses (phase-locked) at twice per input-cycle, respectively. Hence, the LIF system is used as a smart pulse generator that is placed in the feedback signal path for this adaptive sound processing framework. Its transfer function can also be expressed in Laplace form as described in:

$$
\begin{array}{r}
L I F(s)=\left(\frac{K_{p} \cdot e^{-(\tau . d t) \cdot s}}{\tau_{r} \cdot s+1}-\frac{K_{p} \cdot e^{-(W+\tau . d t) \cdot s}}{\tau_{r} \cdot s+1}\right) \\
-\left(\frac{K_{n} \cdot e^{-\left(T_{0}+\tau \cdot d t\right) \cdot s}}{\tau_{r} \cdot s+1}-\frac{K_{p} \cdot e^{-\left(T_{0}+W+\tau \cdot d t\right) \cdot s}}{\tau_{r} \cdot s+1}\right)
\end{array}
$$

where $K_{p}$ represents the amplitude of positive pulses, $K_{n}$ is the amplitude of negative pulses; $T_{0}$ is the time between consecutive pulses (e.g., positive or negative, respectively). For instance, $T_{0}$ can be a value as half of the input signal period (e.g., $T_{0}=T / 2$, where $T$ is the input signal period); $\tau$. $d t$ is the time delay before a pulse being generated (e.g., $\tau$ is the integrator's time constant and $d t$ is the time-step resolution for signal computation); $\tau_{r}$ represents a time constant for the rising of the output signal (i.e., pulses generated by a nonideal driver), and $W$ is the pulse width, as illustrated in Fig. 3 (C).

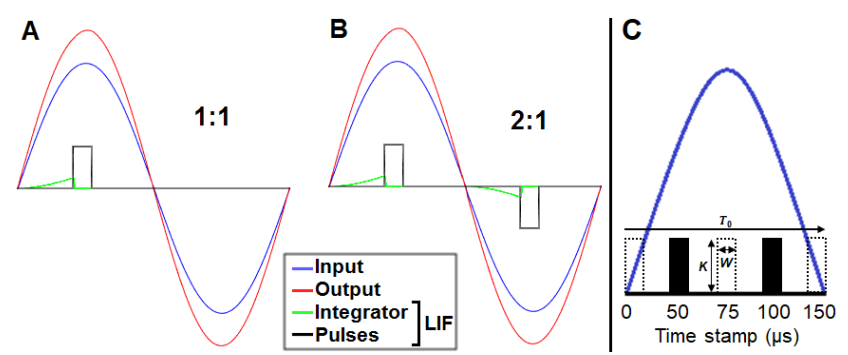

Fig. 3 - Entrainment of pulsatile feedback signals based on 1:1 and 2:1 resonance modes for comparison, (A) and (B), 
respectively; (C) shows the pulse features such as the firing-time within an input-positive-wave-cycle with amplitude $(K)$ and duration $(W)$.

\section{THEORETICAL MODEL APPROACH}

The modelling approach of this feedback system architecture considers the two transfer functions presented in Fig. 2. Therefore, the overall response of this sensory system can be described by the closed-loop transfer function expressed in:

$$
\frac{\operatorname{Output}(s)}{\operatorname{Input}(s)}=\frac{H(s)}{1-\operatorname{LIF}(s) \cdot H(s)}
$$

There is a positive-feedback coupling between the front-end acoustic detector and the back-end computational system, which can result in enhanced and nonlinear output responses. The closed-loop transfer function in (3) can be implemented mathematically following a recursive approach as similarly used in [17]. $H(s)$ can be converted from analog-to-digital domain through a bilinear transformation and computed as an IIR system using a biquadratic topology, whereas, $\operatorname{LIF}(s)$ can be solved according to its time-domain form by applying the implicit Euler method as in [18]. Therefore, pulses are likely to be fired when a threshold level $\left(V_{t h}\right)$ is reached at the integrator's output stage, followed by the system's self-reset period.

\section{RESULTS}

This section presents the simulation results that are used to evaluate the performance of this bio-inspired acoustic concept under some defined input system conditions.

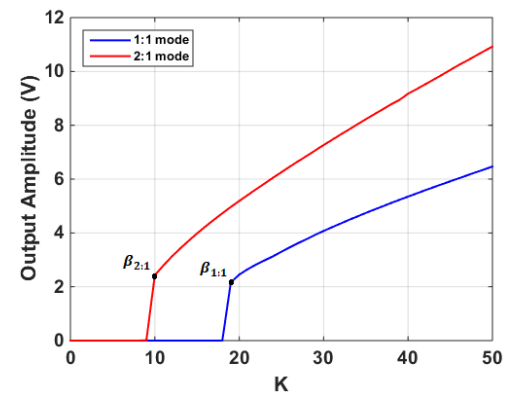

Fig. 4 - Hopf bifurcation of the system while vary the $K$ value used. Comparison between 1:1 and 2:1 modes obtained through simulation based on an impulse response analysis using the following system conditions: $f_{\text {in }}=f_{0}=3.3 \mathrm{kHz}, Q=30, V_{\text {th }}=0.25$, $T_{0}=0.303 \mathrm{~ms}(1: 1)$ and $T_{0}=0.166 \mathrm{~ms}(2: 1), \tau=d t=20 \mu \mathrm{s}, W=$ $20 \mu \mathrm{s}, K_{1: 1}=K_{p}$ and $K_{2: 1}=\left|K_{p}+K_{n}\right|$.

\subsection{Stability}

First of all, the response of this kind of feedback sensor system approach can become unstable under certain conditions. When the system is configured with $2: 1$ mode (e.g., pulses entrained at twice-per-cycle) - this places the critical region/point ( $\beta$ point: it is assumed as the boundary region between a stable operating regime and a criticallystable state of the system - e.g., a self-sustained and continuous oscillatory output response) at the left-side of the 1:1 bifurcation region under the same system conditions, as illustrated in Fig. 4.

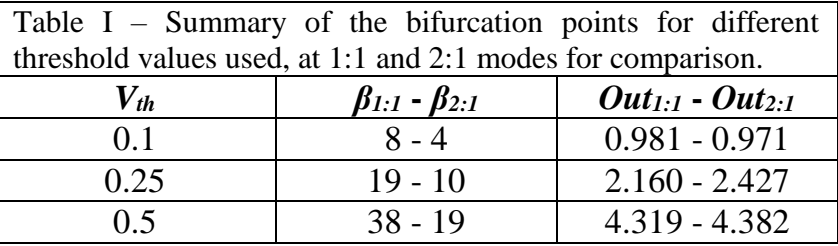

Additionally, Table I provides a summary of the $\beta$ points for different feedback configurations (vary $V_{t h}$ value), where $\beta_{1: 1}$ and $\beta_{2: 1}$ represent the bifurcation points for each resonance mode, respectively; and $O u t_{1: 1}$ and $O u t_{2: 1}$ are the output amplitude levels at which the system transits from a stable to a continuous and oscillatory output response at those particular $\beta$ points, respectively. Refer to [3] for a study when applying Hopf bifurcation can also be used to characterize some hearing responses. Therefore, the overall system's stability diagram is dependent on $V_{t h}, K$ and resonance mode (e.g., 1:1 or $2: 1$ ) chosen.

\subsection{Active Nonlinear Amplification}

Fig. 5 shows three different feedback system configurations such as passive $(K=0), 1: 1\left(K=K_{p}\right)$ and $2: 1\left(K=\left|K_{p}+K_{n}\right|\right)$ resonance modes, for comparison. It is clear that $2: 1$ mode can provide greater signal amplification capabilities given by this sensor system approach when compared with the passive response and also when the 1:1 configuration is used under the same threshold conditions. Note that, the feedback signal ( $K$ amplitude) at 2:1 mode also provides twice the energy per cycle than in 1:1 mode.

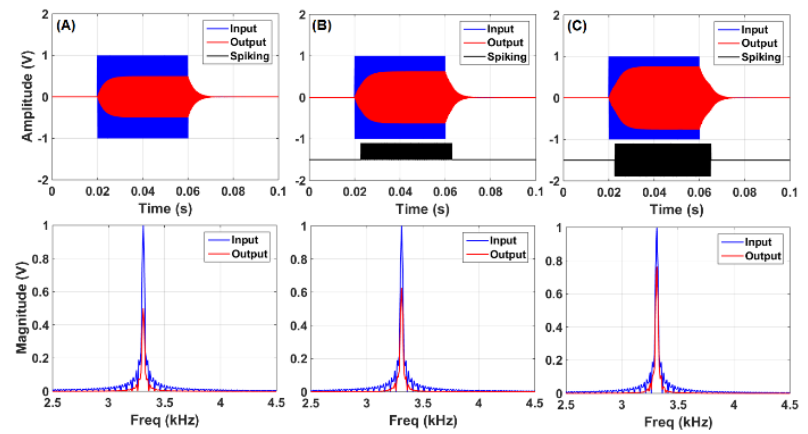

Fig. 5 - Amplification response of the system through simulation using the following system conditions: $f_{\text {in }}=f_{0}=3.3 \mathrm{kHz}, Q=30$, $V_{t h}=0.25, T_{0}=0.303 \mathrm{~ms}(1: 1)$ and $T_{0}=0.166 \mathrm{~ms}(2: 1), \tau=d t=$ $20 \mu \mathrm{s}, W=20 \mu \mathrm{s}$. (A) without feedback signals, hence $K=0$; (B) using 1:1 mode with $K=K_{p}=5$; and (C) using 2:1 mode, hence $K=\left|K_{p}+K_{n}\right|=10$. Blue-trace represents the input sound signal, red-trace is the system's output signal and black-trace represents the phase-locked feedback signals (e.g., square pulses - rescaled for clarity).

It is interesting to note the fact that the feedback system architecture exploited in this study can also exhibit a 
nonlinear input-output relationship such as compressive gain, as illustrated in Fig. 6, (A) using 1:1 and (B) using 2:1, for comparison.

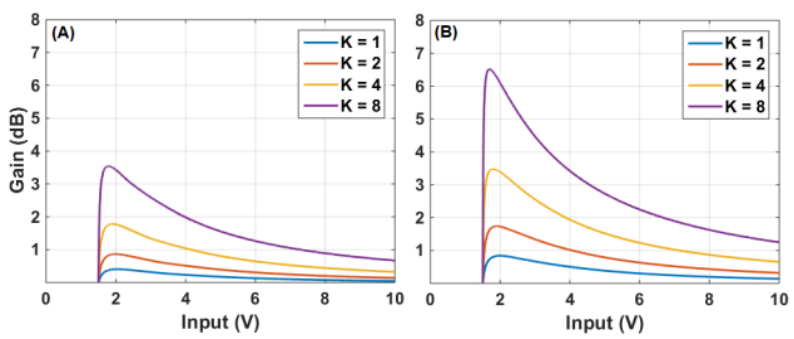

Fig. 6 - Nonlinear compressive gain response of the system using the following conditions: $f_{\text {in }}=f_{0}=3.3 \mathrm{kHz}, Q=30, V_{\text {th }}=0.25, T_{0}$ $=0.303 \mathrm{~ms}(1: 1)$ and $T_{0}=0.166 \mathrm{~ms}(2: 1), \tau=d t=20 \mu \mathrm{s}, W=20$ $\mu \mathrm{s}, K=1,2,4$ and 8 . (A) 1:1 mode and (B) 2:1 mode, for comparison.

\subsection{Hysteresis}

Additionally, this system may also become bistable. That is due to the on-off switching of the feedback signal path, which thus generates a nonlinear behavior of the overall system response such as hysteresis, as presented in Fig. 7. These hysteretic responses are a result of the feedback control operation exploited and depending on $V_{t h}, K$ and resonance mode (e.g., 1:1 or 2:1) defined.

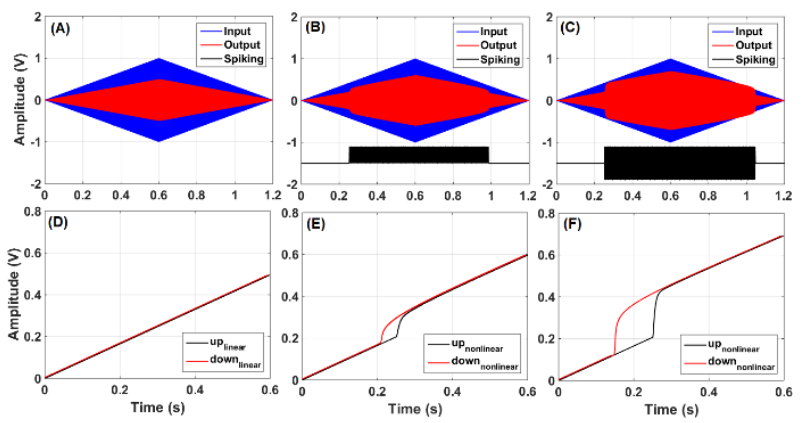

Fig. 7 - Hysteretic output responses of the sensory system can be tested while applying an amplitude modulated input signal. (A) Without feedback operation (e.g., passive response); (B) with feedback operation using 1:1 mode and (C) using 2:1 mode (e.g., active responses), respectively. (D) Linear $v s$ (E) and (F) nonlinear responses of the system, without and with feedback computation, respectively. It faithfully replicate the hysteretic output response presented by the mosquito hearing system reports [11].

\subsection{Intermodulation Products}

Additionally, this feedback control system approach when exposed to competitive input signals may generate intermodulation output responses as a consequence of the nonlinearities in the system, shown for example in Fig. 9, and as summarized in Table II. Typically, intermodulation products are phenomena resulting from nonlinear systems as it has been reported from biological studies related to active hearing processes [3][19] as well. Moreover, these might be used as useful features to identify the presence of signals of interest and perhaps exploited as a signal processing strategy [20].
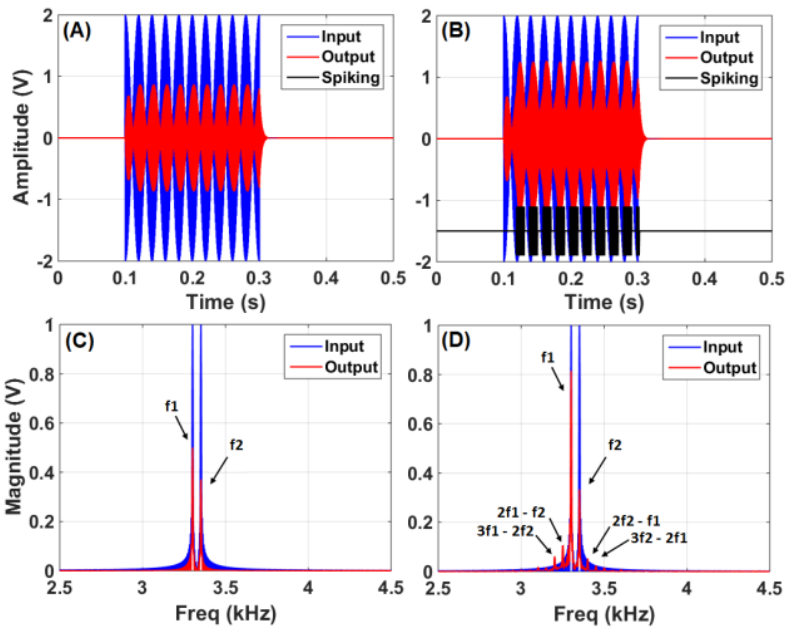

Fig. 9 - Intermodulation output response by this system using the following conditions: $f_{\text {in }}=f_{1}+f_{2} ; f_{1}=f_{0}=3.3 \mathrm{kHz}, f_{2}=3.35 \mathrm{kHz}$; $Q=30, V_{\text {th }}=0.25, K= \pm 6, T_{0}=0.166 \mathrm{~ms}, \tau=d t=20 \mu \mathrm{s}, W=20$ $\mu$ s. (A) and (C) without feedback computation; (B) and (D) with feedback computation, hence intermodulation products appear as a consequence of the nonlinear dynamics in the system.

Table II - Summary of the intermodulation products given by the system for $\Delta f=\left|f_{1}-f_{2}\right|=50 \mathrm{~Hz}$.

\begin{tabular}{|c|c|c|c|c|c|}
\hline$f_{I}$ & $f_{2}$ & $2 f_{1}-f_{2}$ & $3 f_{1}-2 f_{2}$ & $2 f_{2}-f_{1}$ & $3 f_{2}-2 f_{3}$ \\
\hline 3302 & 3352 & 3252 & 3202 & 3402 & 3452 \\
\hline $\mathrm{Hz}$ & $\mathrm{Hz}$ & $\mathrm{Hz}$ & $\mathrm{Hz}$ & $\mathrm{Hz}$ & $\mathrm{Hz}$ \\
\hline
\end{tabular}

\section{CONCLUSION}

This paper presents a theoretical model approach for a propose-built bio-inspired feedback system concept that is simulated numerically using MatLab. A front-end acoustic sensor system can show nonlinear output behavior such as compressive gain, hysteresis and intermodulation products when placed within a positive feedback control mechanism. The 2:1 resonance mode (e.g., entrainment at twice per cycle) can provide greater signal amplification when compared with 1:1 mode and its passive responses, however, it may place the $\beta$ point towards lower values of $K$ and $V_{t h}$ used. This kind of theoretical modelling approach might be useful for better understanding and characterization of feedback control mechanisms applied to acoustic-signal-detection and signal processing that are performed at the transducer level. This kind of signal processing approach may inspire future technological developments as a new type of signal-detection methodology to be applied with potential benefits within acoustic or ultrasonic sensor and system designs.

\section{ACKNOWLEDGEMENTS}

This work was funded by the European Research Council under the European Union's Seventh Framework Programme (FP/20072013) / ERC Grant Agreement n. [615030]. 


\section{REFERENCES}

[1] - C A. Mead, "Neuromorphic electronic system", Proceedings on IEEE, 78(10): 1629-1636, 1990.

[2] - R. Sapershkar, "Analog versus digital: Extrapolated from electronics to neurobiology", Neuronal Computation, 10(7): 1601$1638,1998$.

[3] - A. J. Hudspeth, "Making an effort to listen: Mechanical amplification in the ear", Neuron, 59(4): 530-545, 2008.

[4] - M. Rudnicki, O. Schoppe, M. Isik, F. Volk, and W. Hemmert, "Modeling auditory coding: from sound to spikes", Springer, Cell Tissue Res., 361: 159-175, 2015.

[5] - R. F. Lyon, "Human and Machine Hearing: Extracting Meaning from Sound", Cambridge University Press, 2018.

[6] - T. Rodriguez and R. Garcia, "Theory of Q control in atomic force microscopy", Applied Physics Letters, 82(26): 4821-4823, 2003.

[7] - G. Prakash, S. Hu, A. Raman, and R. Reifenberger, "Theoretical basis of parametric-resonance-based atomic force microscopy”, Phys. Rev. B, 79, 094304, 2009.

[8] - J. Guerreiro, J. C. Jackson, and J. F. C. Windmill, "Simple Ears Inspire Frequency Agility in an Engineered Acoustic Sensor System," IEEE Sensors Journal, 17(22): 7298-7305, 2017.

[9] - K. Dierkes, B. Lindner, and F. Jülicher, "Enhancement of sensitivity gain and frequency tuning by coupling of active hair bundles," PNAS, 105(48): 18669-18674, 2008.

[10] - N. Mhatre and D. Robert, "A tympanal insect ear exploits a critical oscillator for active amplification and tuning," Cell Press, Current Biology, 23(19): 1952-1957, 2013.

[11] - J. C. Jackson, J. F. C. Windmill, V. G. Pook, and D. Robert, "Synchrony through twice-frequency forcing for sensitive and selective auditory processing," PNAS, 106(25): 10177-10182, 2009.

[12] - A. Pikovsky, M. Rosenblum, and J. Kurths, "Synchronization: A Universal Concept in Nonlinear Sciences." Cambridge Univ. Press, 2001.

[13] - J. F. C. Windmill and J. C. Jackson, "Mechanical Specializations of Insect Ears. In: Insect Hearing", Springer Handbook of Auditory Research, 2016.

[14] - A. A. Lazar, "Time encoding with an integrate-and-fire neuron with a refractory period," Neurocomputing, vol. 58 , no. 60 , pp. 53-58, 2004.

[15] - A. S. Alvarado, M. Rastogi, J. G. Harris, and J. Principe, "The integrate-and-fire sampler: A special type of asynchronous $\Sigma$ - $\Delta$ modulator," in Proc. IEEE Int. Sym. Circuits Syst., 2011.

[16] - J. Guerreiro, A. Reid, J. C. Jackson and J. F. C. Windmill, "Bio-inspired Active Amplification in a MEMS Microphone using
Feedback Computation", Proceedings on IEEE Biomedical Circuits and Systems Conference, 2017;

[17] - J. Guerreiro, A. Reid, J. C. Jackson, and J. F. C. Windmill, "Active hearing mechanisms inspire adaptive amplification in an acoustic sensor system," IEEE Transactions on Biomedical Circuits and Systems, 12(3): 655-664, 2018.

[18] - M. J. Skocik and L. N. Long, "On the capabilities and computational costs of neuron models," IEEE Trans. Neural Netw. Learn. Syst., 25(8): 1474-1483, 2014.

[19] - T. A. J. Duke and F. Jülicher, "Critical oscillators as active elements in hearing. in: Active processes and otoacoustic emissions in hearing," Springer Handbook of Auditory Research, 30: 63-92, 2007.

[20] - P. M. Simoes, R. A. Ingham, G. Gibson, and I. J. Russel, “A role for acoustic distortion in novel rapid frequency modulation behaviour in freeflying male mosquitoes," Journal of Experimental Biology, 219: 2039 - 2047, 2016. 